\title{
THE VARIETY GENERATED BY ALL MONOIDS OF ORDER FOUR IS FINITELY BASED
}

\author{
Edmond W. H. LeE AND JiAn Rong Li ${ }^{1}$ \\ Nova Southeastern University, USA and Lanzhou University, China \\ Dedicated to Professor Mikhail V. Volkov on the occasion of his 60th birthday
}

\begin{abstract}
It is known that the variety $\mathbf{M}_{n}$ generated by all monoids of order $n$ is finitely based if $n \leq 3$ and non-finitely based if $n \geq 6$. The present article establishes the finite basis property of the variety $\mathbf{M}_{4}$. This leaves $\mathbf{M}_{5}$ as the last open case in the finite basis problem for the varieties $\mathbf{M}_{n}$.
\end{abstract}

\section{InTRODUCTION}

1.1. Finite basis problem. A basis for a semigroup $\mathcal{S}$ is a set of identities satisfied by $\mathcal{S}$ that axiomatizes all identities of $\mathcal{S}$. A semigroup is finitely based if it has a finite basis. By 1970, the finite basis property has been established for several important classes of semigroups such as commutative semigroups $([18])$, idempotent semigroups $([2,6,7])$, and finite groups $([17])$. However, examples of non-finitely based finite semigroups ([18]) have also been discovered by then, and presently, the problem of deciding when a finite semigroup is finitely based remains open. This led researchers to investigate, over the years, the finite basis problem for various explicit classes of semigroups that either exist very naturally or play important roles in other parts of mathematics. Some of these examples include the semigroup $\mathcal{B}_{n}$ of binary relations on $\{1,2, \ldots, n\}$ and its subsemigroup $\mathcal{R}_{n}$ of reflexive binary relations, and the

2010 Mathematics Subject Classification. 20M07.

Key words and phrases. Monoid, semigroup, variety, finitely based.

${ }^{1}$ Corresponding author.

The second author is supported by the National Natural Science Foundation of China (no. 11501267, 11371177, 11401275) and the Fundamental Research Funds for the Central Universities of China (no. lzujbky-2015-78). 
semigroup $\mathcal{T}_{n}$ of transformations of $\{1,2, \ldots, n\}$ and its subsemigroup $\mathcal{E}_{n}$ of extensive transformations. These semigroups are non-finitely based for all except the first few values of $n$ :

- $\mathcal{B}_{n}$ is finitely based if and only if $n=1$ ([23]);

- $\mathcal{T}_{n}$ is finitely based if and only if $n \leq 2$ ([23]);

- $\mathcal{R}_{n}$ and $\mathcal{E}_{n}$ are finitely based if and only if $n \leq 4([9,14,25])$.

Refer to the surveys by Shevrin and Volkov ([20]) and Volkov ([24]) for more information on the finite basis problem for finite semigroups in general.

1.2. Main result. The finite basis property of finite semigroups has also been investigated collectively. For each $n \geq 1$, let $\mathbf{S}_{n}$ denote the variety generated by all semigroups of order $n$ and let $\mathbf{M}_{n}$ denote the variety generated by all monoids of order $n$. In the 1980s, Volkov ([23]) considered the variety $\mathbf{S}_{n}$ and proved that it is non-finitely based whenever $n \geq 5$. In fact, since the Brandt monoid of order six is well known to be inherently non-finitely based ([19]), the varieties $\mathbf{S}_{n}$ and $\mathbf{M}_{n}$ are non-finitely based for all $n \geq 6$. On the other hand, it follows from Luo and Zhang ([16]) that the varieties $\mathbf{S}_{n}$ and $\mathbf{M}_{n}$ are finitely based if $n \leq 3$. Recently, Li et al. ([15]) proved that the variety $\mathbf{S}_{4}$ is finitely based. Consequently, the variety $\mathbf{S}_{n}$ is finitely based if and only if $n \leq 4$. The finite basis property of the varieties $\mathbf{M}_{4}$ and $\mathbf{M}_{5}$ currently remains unknown.

The objective of the present article is to prove that the variety $\mathbf{M}_{4}$ is finitely based. To simplify the statement of the main result, define the deletion closure of an identity $U \approx V$, written $U \stackrel{*}{\approx} V$, to be the identity system that contains $U \approx V$ and any nontrivial identity obtained by eliminating all occurrences of some letters in $U \approx V$. For instance, the deletion closure $x h x k x^{2} \stackrel{*}{\approx} h x^{2} k x$ represents the system

$$
x h x k x^{2} \approx h x^{2} k x, \quad x h x^{3} \approx h x^{3}, \quad x^{2} k x^{2} \approx x^{2} k x, \quad x^{4} \approx x^{3} .
$$

Note that if a monoid satisfies an identity $U \approx V$, then it also satisfies the deletion closure $U \stackrel{*}{\approx} V$.

\section{ThEOREM 1.1. The identities}

$$
\begin{gathered}
x^{13} h x k x \stackrel{*}{\approx} x h x k x, \quad x h x^{2} k x \stackrel{*}{\approx} x^{3} h k x, \quad x h y^{2} x^{2} k y \stackrel{*}{\approx} x h x^{2} y^{2} k y, \\
x h y k x y t x d y \stackrel{*}{\approx} x h y k y x t x d y, \quad \text { xhykxytydx} \stackrel{*}{\approx} x h y k y x t y d x
\end{gathered}
$$

constitute a finite basis for the variety $\mathbf{M}_{4}$.

The finite basis property of the varieties $\mathbf{S}_{n}$ and $\mathbf{M}_{n}$, with the exception of the open case $\mathbf{M}_{5}$, is summarized in the following table:

\begin{tabular}{rccc}
\hline & $n \leq 4$ & $n=5$ & $n \geq 6$ \\
\hline $\mathbf{S}_{n}$ is finitely based & Yes & No & No \\
$\mathbf{M}_{n}$ is finitely based & Yes & $?$ & No \\
\hline
\end{tabular}


It is of interest to note that while semigroups of order five are all finitely based $([11,22])$, the variety $\mathbf{S}_{5}$ they generate is not.

1.3. Hereditarily finitely based varieties. A finitely based variety that satisfies the stronger property of containing only finitely based semigroups is said to be hereditarily finitely based. Since each finite basis involves finitely many identities with finitely many variables, membership in a hereditarily finitely based variety provides a sufficient condition for the finite basis property that can be verified in polynomial time. When identifying finitely based semigroups from a given fixed class $\mathfrak{C}$, one obvious first step is to find out which semigroups from $\mathfrak{C}$ belong to known hereditarily finitely based varieties. For instance, this technique was employed in the classification of all finitely based semigroups of order six $([12,13])$.

Recently, Luo and Zhang proved that the variety $\mathbf{S}_{3}$ is hereditarily finitely based ([16]); the varieties $\mathbf{S}_{n}$ and $\mathbf{M}_{n}$ thus satisfy the same property whenever $n \leq 3$. Since the varieties $\mathbf{S}_{4}$ and $\mathbf{M}_{4}$ are finitely based, it is natural to question if they are also hereditarily finite based. The answer to this question is, however, known for some time to be negative ([8, Corollary 3.17]). It follows that the finite basis property of the varieties $\mathbf{S}_{4}$ and $\mathbf{M}_{4}$ cannot be deduced by recognizing them as subvarieties of some hereditarily finitely based varieties. Exhibiting explicit finite bases seems unavoidable in establishing the finite basis property for $\mathbf{S}_{4}$ and $\mathbf{M}_{4}$; the same situation likely applies to the open case $\mathbf{M}_{5}$ if it turns out to be finitely based.

1.4. Organization. After some background material is given in Section 2, it is shown in Section 3 that the variety $\mathbf{M}_{4}$ is generated by only five monoids of order at most four. This enables one to easily verify that the variety $\mathbf{M}_{4}$ satisfies the identities (0). Restrictions on identities satisfied by the variety $\mathbf{M}_{4}$ are established in Section 4; these results are then employed in Section 5 to complete the proof of Theorem 1.1.

\section{Preliminaries}

The following semigroups are required in the present article, especially in giving a simple generating set for the variety $\mathbf{M}_{4}$ :

- the cyclic group $\mathcal{C}_{n}=\left\langle a \mid a^{n}=1\right\rangle$ of order $n$;

- the nilpotent semigroup $\mathcal{N}_{n}=\left\langle a \mid a^{n+1}=a^{n}\right\rangle$ of order $n$;

- the semigroup $\mathcal{T}_{2}=\{\alpha, \beta, \gamma, \epsilon\}$ of transformations of $\{1,2\}$, where

$1 \alpha=2 \alpha=1, \quad 1 \beta=2 \beta=2, \quad 1 \gamma=2,2 \gamma=1, \quad$ and $\quad 1 \epsilon=1,2 \epsilon=2$;

- the semigroup $\mathcal{T}_{2}^{\nabla}$ anti-isomorphic to $\mathcal{T}_{2}$.

For any semigroup $\mathcal{S}$, let $\mathcal{S}^{1}$ denote the monoid obtained by adjoining a unit element to $\mathcal{S}$. 
2.1. Identities and bases. Let $\Sigma^{+}$and $\Sigma^{*}$ respectively denote the free semigroup and free monoid over a countably infinite alphabet $\Sigma$. Elements of $\Sigma$ are called letters and elements of $\Sigma^{*}$ are called words. An identity is written as $U \approx V$ where $U, V \in \Sigma^{+}$. A semigroup $\mathcal{S}$ satisfies an identity $U \approx V$ if, for any substitution $\varphi: \Sigma \rightarrow \mathcal{S}$, the elements $U \varphi$ and $V \varphi$ of $\mathcal{S}$ coincide. A set $\Theta$ of identities satisfied by a semigroup $\mathcal{S}$ is a basis for $\mathcal{S}$ if $\Theta$ implies every identity satisfied by $\mathcal{S}$. A semigroup is finitely based if it possesses a finite basis.

2.2. Content and occurrence. The content of a word $U$, denoted by $\operatorname{con}(U)$, is the set of letters occurring in $U$. The number of occurrences of a letter $x$ in a word $U$ is denoted by $\operatorname{occ}(x, U)$. The length of a word $U$ is thus

$$
|U|=\sum\{\operatorname{occ}(x, U) \mid x \in \operatorname{con}(U)\} .
$$

For example, if $U=x^{2} z x y^{2} x t z x^{3} t^{4}$, then $\operatorname{con}(U)=\{x, y, z, t\}, \operatorname{occ}(x, U)=7$, $\operatorname{occ}(y, U)=\operatorname{occ}(z, U)=2 \operatorname{occ}(t, U)=5$, and $|U|=7+2+2+5=16$.

LEMMA 2.1 ([1, Lemma 6.1.4]). Let $U \approx V$ be any identity satisfied by the monoid $\mathcal{N}_{3}^{1}$. Then for each $x \in \Sigma$, precisely one of the following holds:

(i) $\operatorname{occ}(x, U)=\operatorname{occ}(x, V) \leq 2$;

(ii) $\operatorname{occ}(x, U), \operatorname{occ}(x, V) \geq \overline{3}$.

Consequently, $\operatorname{con}(U)=\operatorname{con}(V)$.

LEMMA 2.2. Let $U \approx V$ be any identity satisfied by both the groups $\mathcal{C}_{3}$ and $\mathcal{C}_{4}$. Then $\operatorname{occ}(x, U) \equiv \operatorname{occ}(x, V)(\bmod 12)$ for all $x \in \Sigma$. Consequently, $\operatorname{occ}(x, U) \equiv \operatorname{occ}(x, V)(\bmod 2)$ for all $x \in \Sigma$.

Proof. It is well known and easily verified that if $U \approx V$ is any identity satisfied by the group $\mathcal{C}_{n}$, then $\operatorname{occ}(x, U) \equiv \operatorname{occ}(x, V)(\bmod n)$ for all $x \in \Sigma$. The result then follows because $\mathcal{C}_{12} \cong \mathcal{C}_{3} \times \mathcal{C}_{4}$.

2.3. Initial part, final part, and precedence. The initial part of a word $U$, denoted by $\operatorname{ini}(U)$, is the word obtained by retaining the first occurrence of each letter in $U$. The final part of a word $U$, denoted by $\operatorname{fin}(U)$, is the word obtained by retaining the last occurrence of each letter in $U$. For example, if $U=x^{2} z x y^{2} x t z x^{3} t^{4}$, then $\operatorname{ini}(U)=x z y t$ and $\operatorname{fin}(U)=y z x t$.

For any distinct letters $x$ and $y$ of a word $U$, let $\operatorname{occ}(\dot{x}, y, U)$ denote the number of occurrences of $x$ preceding the first occurrence of $y$, and let $\operatorname{occ}(y, \dot{x}, U)$ denote the number of occurrences of $x$ following the last occurrence of $y$. In particular,

- if $m=\operatorname{occ}(\dot{x}, y, U)$, then

$$
U=A_{0} x A_{1} x A_{2} \cdots x A_{m} y B
$$

for some $A_{0}, A_{1}, \ldots, A_{m}, B \in \Sigma^{*}$ such that $x, y \notin \operatorname{con}\left(A_{0} A_{1} \cdots A_{m}\right)$; 
- if $m=\operatorname{occ}(x, \dot{y}, U)$, then

$$
U=A x B_{0} y B_{1} y B_{2} \cdots y B_{m}
$$

for some $A, B_{0}, B_{1}, \ldots, B_{m} \in \Sigma^{*}$ such that $x, y \notin \operatorname{con}\left(B_{0} B_{1} \cdots B_{m}\right)$.

Lemma 2.3. Let $U \approx V$ be any identity satisfied by both the monoids $\mathcal{T}_{2}$ and $\mathcal{T}_{2}^{\nabla}$. Then

(i) $\operatorname{ini}(U)=\operatorname{ini}(V)$;

(ii) $\operatorname{fin}(U)=\operatorname{fin}(V)$;

(iii) $\operatorname{occ}(\dot{x}, y, U) \equiv \operatorname{occ}(\dot{x}, y, V)(\bmod 2)$ for all $x, y \in \Sigma$;

(iv) $\operatorname{occ}(y, \dot{x}, U) \equiv \operatorname{occ}(y, \dot{x}, V)(\bmod 2)$ for all $x, y \in \Sigma$.

Proof. Parts (i) and (iii) follow from Edmunds [4, Lemma 4.5], while parts (ii) and (iv) hold by symmetry.

\section{A small set of Generators For $\mathbf{M}_{4}$}

In this section, let

$$
\mathfrak{M}=\left\{\mathcal{C}_{3}, \mathcal{C}_{4}, \mathcal{N}_{3}^{1}, \mathcal{T}_{2}, \mathcal{T}_{2}^{\nabla}\right\}
$$

For any class $\mathfrak{C}$ of semigroups, let var $\mathfrak{C}$ denote the variety generated by $\mathfrak{C}$.

Proposition 3.1. $\mathbf{M}_{4}=\operatorname{var} \mathfrak{M}$.

Corollary 3.2. The variety $\mathbf{M}_{4}$ satisfies the identities (0).

Proof. It is routinely checked that the monoids in $\mathfrak{M}$ satisfy the identities (0). The result then follows from Proposition 3.1.

LEMMA 3.3. Any semigroup that satisfies any of the following identity systems belongs to the variety var $\mathfrak{M}$ :

$$
\begin{gathered}
x^{15} \approx x^{3}, \quad x y \approx y x ; \\
x^{3} \approx x^{2}, \quad x y x \approx x^{2} y ; \\
x^{3} \approx x^{2}, \quad x y x \approx y x^{2} ; \\
x^{3} \approx x, \quad x y x^{2} \approx x y, \quad x y x y \approx x y^{2} x ; \\
x^{3} \approx x, \quad x^{2} y x \approx y x, \quad x y x y \approx y x^{2} y .
\end{gathered}
$$

Proof. It suffices to show that each of the five identity systems defines a subvariety of var $\mathfrak{M}$.

The identities (3.1) define the variety $\operatorname{var}\left\{\mathcal{D}^{1}\right\}$, where $\mathcal{D}=\left\langle a \mid a^{15}=a^{3}\right\rangle$; see Almeida [1, Corollary 6.1.5]. Since the monoid $\mathcal{D}^{1}$ is isomorphic to the submonoid of $\mathcal{C}_{3} \times \mathcal{C}_{4} \times \mathcal{N}_{3}^{1}$ generated by the element $(a, a, a)$, the inclusion $\operatorname{var}\left\{\mathcal{D}^{1}\right\} \subseteq \operatorname{var} \mathfrak{M}$ holds.

The identities (3.2) define the variety $\operatorname{var}\left\{\mathcal{N}_{2}^{1}, \mathcal{L}_{2}^{1}\right\}$, where $\mathcal{L}_{2}$ is the left-zero semigroup of order two; see Lee [10, Lemma 3.3(iv)]. Since the monoids $\mathcal{N}_{2}^{1}$ and $\mathcal{L}_{2}^{1}$ are isomorphic to submonoids of $\mathcal{N}_{3}^{1}$ and $\mathcal{T}_{2}^{\nabla}$ respectively, 
the inclusion $\operatorname{var}\left\{\mathcal{N}_{2}^{1}, \mathcal{L}_{2}^{1}\right\} \subseteq \operatorname{var} \mathfrak{M}$ holds. By symmetry, the identities (3.3) also define a subvariety of var $\mathfrak{M}$.

The identities (3.4) define the variety $\operatorname{var}\left\{\mathcal{T}_{2}^{\nabla}\right\}$ ([5, part 9 of the first proposition]), which is obviously a subvariety of var $\mathfrak{M}$. By symmetry, the identities (3.5) also define a subvariety of var $\mathfrak{M}$.

Up to isomorphism, there exist 35 monoids of order four ([21]). Multiplication tables of these 35 monoids are lexicographically listed below; the underlying set of each monoid is $\{1,2,3,4\}$, and each table is given by a $4 \times 4$ array where the $(i, j)$-entry denotes the product of the elements $i$ and $j$ :

$\begin{array}{llllllllll}1111 & 1111 & 1111 & 1111 & 1111 & 1111 & 1111 & 1111 & 1111 & 1111 \\ 1112 & 1112 & 1112 & 1112 & 1112 & 1122 & 1122 & 1122 & 1212 & 1212 \\ 1113 & 1123 & 1133 & 1233 & 3333 & 1133 & 1233 & 1234 & 1133 & 3333 \\ 1234 & 1234 & 1234 & 1234 & 1234 & 1234 & 1234 & 1243 & 1234 & 1234 \\ (3.1) & (3.1) & (3.1) & (3.3) & (3.2) & (3.2) & (3.1) & (3.1) & (3.1) & (3.2) \\ 1111 & 1111 & 1111 & 1111 & 1111 & 1111 & 1111 & 1111 & 1111 & 1111 \\ 1222 & 1222 & 1222 & 1222 & 1224 & 1224 & 1224 & 1234 & 1234 & 1234 \\ 1223 & 1233 & 1234 & 1234 & 1234 & 1234 & 1234 & 1324 & 1342 & 3333 \\ 1234 & 1234 & 1243 & 1444 & 1244 & 1442 & 4444 & 4444 & 1423 & 3412 \\ (3.1) & (3.1) & (3.1) & (3.2) & (3.3) & (3.1) & (3.2) & (3.4) & (3.1) & (3.4) \\ 1111 & 1114 & 1114 & 1114 & 1114 & 1114 & 1114 & 1114 & 1133 & 1133 \\ 1234 & 1124 & 1124 & 1224 & 1224 & 1224 & 1234 & 1234 & 1234 & 1234 \\ 3333 & 1234 & 1234 & 1234 & 1234 & 1234 & 1324 & 1324 & 1331 & 3311 \\ 4444 & 1144 & 4441 & 1144 & 1444 & 4441 & 1444 & 4441 & 1432 & 3411 \\ (3.2) & (3.3) & (3.1) & (3.3) & (3.3) & (3.1) & (3.5) & (3.1) & (3.5) & (3.1) \\ 1133 & 1134 & 1134 & 1234 & 1234 & & & & & \\ 1234 & 1234 & 1234 & 2143 & 2143 & & & & & \\ 3311 & 1334 & 3341 & 3412 & 3421 & & & & & \\ 3412 & 1434 & 4413 & 4321 & 4312 & & & & & \\ (3.1) & (3.3) & (3.1) & (3.1) & (3.1) & & & & & \end{array}$

Below each table is listed an identity system from Lemma 3.3 that is satisfied by the monoid. Therefore the inclusion $\mathbf{M}_{4} \subseteq$ var $\mathfrak{M}$ holds. The inclusion $\operatorname{var} \mathfrak{M} \subseteq \mathbf{M}_{4}$ is obvious because each monoid in $\mathfrak{M}$ is of order at most four. In particular, if $\mathcal{A}_{n}$ denotes the $n$-th monoid from above, then the monoids

$$
\mathcal{A}_{2} \cong \mathcal{N}_{3}^{1}, \quad \mathcal{A}_{20} \cong \mathcal{T}_{2}^{\nabla}, \quad \mathcal{A}_{29} \cong \mathcal{T}_{2}, \quad \mathcal{A}_{33} \cong \mathcal{C}_{3}^{1}, \quad \text { and } \quad \mathcal{A}_{35} \cong \mathcal{C}_{4}
$$

generate the variety $\mathbf{M}_{4}$.

\section{IDENTITIES FORMED BY WORDS IN CANONICAL FORM}

4.1. Identities satisfied by $\mathbf{M}_{4}$. The following lemma, the proof of which is routine, contains some useful identities deducible from the identities (0).

LEMMA 4.1. The identities (0) imply the identities

$$
\begin{gathered}
x h x^{12} k x t x \stackrel{*}{\approx} x h k x t x, \quad x h x k x^{12} t x \stackrel{*}{\approx} x h x k t x, \\
x h x^{2} k x \approx x^{3} h k x, \quad x^{3} h x \approx x h x^{3},
\end{gathered}
$$




$$
\begin{gathered}
x h y^{2} x^{2} k y \stackrel{*}{\approx} x h x^{2} y^{2} k y, \\
x h y k x y t x d y \stackrel{*}{\approx} x h y k y x t x d y, \quad \text { xhykxytydx} \stackrel{*}{\approx} x h y k y x t y d x .
\end{gathered}
$$

LEMMA 4.2. The identities (4.1) imply the following identities:

$$
A x B \cdot y^{2 n} C x^{2 m} \cdot D y E \approx A x B \cdot C x^{2 m} C^{11} y^{2 n} C \cdot D y E
$$

for any $m, n \geq 1$ and $A, B, C, D, E \in \Sigma^{*}$ with $\operatorname{con}(C) \subseteq \operatorname{con}(A B) \cap \operatorname{con}(D E)$.

Proof. There are two cases depending on the values of $m$ and $n$. CASE 1: $m=n=1$. The following is established by induction on $k \geq 0$ :

$\left(\dagger_{k}\right)$ If $|C|=k$, then the identities (4.1) imply the identity (4.2).

If $k=0$, then the identity (4.1c) clearly implies the identity (4.2). Hence $\left(\dagger_{0}\right)$ holds. Suppose that $\left(\dagger_{k}\right)$ holds for some $k>0$. Let $|C|=k+1$, so that $C=z Z$ for some $z \in \Sigma$ and $Z \in \Sigma^{+}$with $|Z|=k$. Then $\left(\dagger_{k+1}\right)$ holds because

$$
\begin{aligned}
A x B \cdot y^{2} C x^{2} \cdot D y E & =A x B \cdot y^{2} z Z x^{2} \cdot D y E \\
& \stackrel{(4.1 \mathrm{~d})}{\approx} A x B \cdot y z y Z x^{2} \cdot D y E \\
& \stackrel{(4.1 \mathrm{a})}{\approx} A x B \cdot z^{12} y z y Z x^{2} z^{12} \cdot D y E \\
& \stackrel{(4.1 \mathrm{a})}{\approx} A x B \cdot z^{12} y z y Z x^{2} z y^{12} z^{11} \cdot D y E \\
& =A x B \cdot z^{11}(z y)^{2} Z x^{2}(z y) y^{11} z^{11} \cdot D y E \\
& \stackrel{(4.2)}{\approx} A x B \cdot z^{11} Z x^{2} Z^{11}(z y)^{2} Z(z y) y^{11} z^{11} \cdot D y E \\
& \stackrel{(4.1 \mathrm{~d})}{ } \\
& \left({ }^{4.1 \mathrm{a})} A x B \cdot z^{11} Z x^{2} z Z^{11} y^{2} z Z y^{12} z^{12} \cdot D y E\right. \\
& \stackrel{(4.1 \mathrm{~b})}{ } A x B \cdot z^{11} Z x^{2} z Z^{11} y^{2} z Z \cdot D y E \\
& \stackrel{(4.1 \mathrm{~d})}{ } A x B \cdot z Z x^{2} z^{11} Z^{11} y^{2} z Z \cdot D y E \\
& \approx x B \cdot z Z x^{2}(z Z)^{11} y^{2} z Z \cdot D y E \\
& =A x B \cdot C x^{2} C^{11} y^{2} C \cdot D y E .
\end{aligned}
$$

CASE $2: m, n \geq 1$. Then

$$
\begin{aligned}
A x B \cdot y^{2 n} C x^{2 m} \cdot D y E & \stackrel{(4.1 \mathrm{~b})}{\approx} A x^{2 m-1} B \cdot y^{2} C x^{2} \cdot D y^{2 n-1} E \\
& \stackrel{(4.2)}{\approx} A x^{2 m-1} B \cdot C x^{2} C^{11} y^{2} C \cdot D y^{2 n-1} E \\
& \stackrel{(4.1 b)}{\approx} A x B \cdot C x^{2 m} C^{11} y^{2 n} C \cdot D y E,
\end{aligned}
$$

where the second deduction holds by Case 1 . 
4.2. Stacks. Suppose that a word $U$ can be written in the form

$$
U=U_{0} \prod_{i=1}^{m}\left(x^{e_{i}} U_{i}\right)=U_{0} x^{e_{1}} U_{1} \cdots x^{e_{m}} U_{m},
$$

where $x \in \Sigma, e_{1}, e_{2}, \ldots, e_{m} \geq 1, U_{0}, U_{m} \in \Sigma^{*}$, and $U_{1}, U_{2}, \ldots, U_{m-1} \in \Sigma^{+}$are such that $x \notin \operatorname{con}\left(U_{0} U_{1} \cdots U_{m}\right)$. Then

- the factors $x^{e_{1}}, x^{e_{2}}, \ldots, x^{e_{m}}$ are called $x$-stacks, or simply stacks, of $U$;

- the first $x$-stack $x^{e_{1}}$ and the last $x$-stack $x^{e_{m}}$ are collectively called exterior $x$-stacks of $U$;

- the non-exterior $x$-stacks $x^{e_{2}}, x^{e_{3}}, \ldots, x^{e_{m-1}}$ are called interior $x$-stacks of $U$;

- $e_{i}$ is the exponent of the stack $x^{e_{i}}$.

Note that if $m \in\{1,2\}$ in (4.3), then $U$ has no interior $x$-stacks. If $m=1$ in (4.3), then $U=U_{0} x^{e_{1}} U_{1}$, so that the first $x$-stack coincides with the last $x$-stack; in this case, $x^{e_{1}}$ is also called a lone stack of $U$.

4.3. Exterior vectors. The exterior vector of a word $U$, denoted by $\operatorname{ext}(U)$, is the vector with the exterior stacks of $U$ as its components. For instance, if

$$
\begin{aligned}
U & =x^{3} \cdot y \cdot x^{2} \cdot z^{5} \cdot y \cdot t \cdot y^{4} \cdot x \cdot y^{2} \cdot z^{2} \cdot y \\
& =A \cdot B \cdot C \cdot D \cdot E \cdot F \cdot G \cdot H \cdot I \cdot J \cdot K,
\end{aligned}
$$

then

$$
\operatorname{ext}(U)=(A, B, D, F, H, J, K)=\left(x^{3}, y, z^{5}, t, x, z^{2}, y\right) .
$$

Note that since each entry of $\operatorname{ext}(U)$ is an exterior stack of $U$, no more than two entries in $\operatorname{ext}(U)$ share the same letter.

The separation degree of a letter $x$ in a word $U$, denoted by $\operatorname{sep}(x, U)$, is the number of exterior stacks of $U$ that occur between two exterior $x$-stacks of $U$. If $x^{e}$ is a lone stack of $U$, then define $\operatorname{sep}(x, U)=0$. The separation degree of a word $U$ is the number

$$
\operatorname{sep}(U)=\sum\{\operatorname{sep}(x, U) \mid x \in \operatorname{con}(U)\} .
$$

EXAMPLE 4.3. For the word $U$ in (4.4),

(i) $\operatorname{sep}(x, U)=|\{B, D, F\}|=3$;

(ii) $\operatorname{sep}(y, U)=|\{D, F, H, J\}|=4$;

(iii) $\operatorname{sep}(z, U)=|\{F, H\}|=2$;

(iv) $\operatorname{sep}(t, U)=0$;

(v) $\operatorname{sep}(U)=3+4+2+0=9$.

4.4. Canonical form. A word $U$ that contains at least two distinct letters is said to be in canonical form if

$$
U=x_{0}^{e_{0}} \prod_{i=1}^{m}\left(U_{i} x_{i}^{e_{i}}\right),
$$


where $x_{0}, x_{1}, \ldots, x_{m} \in \Sigma, e_{0}, e_{1}, \ldots, e_{m} \geq 1$, and $U_{1}, U_{2}, \ldots, U_{m} \in \Sigma^{*}$ satisfy all of the following:

$(\mathrm{CF} 1) \operatorname{ext}(U)=\left(x_{0}^{e_{0}}, x_{1}^{e_{1}}, \ldots, x_{m}^{e_{m}}\right)$, so that $\operatorname{con}(U)=\bigcup_{i=0}^{m}\left\{x_{i}\right\}$;

(CF2) ext $(U)$ is not of the form $\left(\ldots, x^{p}, \ldots, y^{q}, x^{r}, \ldots, y^{s}, \ldots\right)$ with $q$ and $r$ even;

(CF3) the letters of $U_{i}$ are in strict alphabetical order with $x_{i-1}, x_{i} \notin \operatorname{con}\left(U_{i}\right)$;

(CF4) if $x_{i-1}=x_{i}$, then $U_{i} \neq \emptyset$ and $e_{i-1}=1$;

(CF5) (a) if $x_{i}^{e_{i}}$ is a lone stack of $U$, then $e_{i} \leq 14$;

(b) if $x_{i}^{e_{i}}$ is a non-lone first stack of $U$, then $e_{i} \leq 2$;

(c) if $x_{i}^{e_{i}}$ is a non-lone last stack of $U$ and $\operatorname{occ}\left(x_{i}, U\right)=e_{i}+1$, then $e_{i} \leq 13$

(d) if $x_{i}^{e_{i}}$ is a non-lone last stack of $U$ and $\operatorname{occ}\left(x_{i}, U\right) \geq e_{i}+2$, then $e_{i} \leq 12$

REMARK 4.4. If $U_{i}=\emptyset$, then $x_{i-1} \neq x_{i}$ because $x_{i-1}^{e_{i-1}}$ and $x_{i}^{e_{i}}$ are stacks. Since $U_{1}=U_{m}=\emptyset$ by (CF3), it follows that $x_{0} \neq x_{1}$ and $x_{m-1} \neq x_{m}$. In particular,

(i) if $m=1$, then $U=x_{0}^{e_{0}} x_{1}^{e_{1}}$ where $x_{0}^{e_{0}}$ and $x_{1}^{e_{1}}$ are lone stacks;

(ii) if $m=2$, then $U=x_{0}^{e_{0}} x_{1}^{e_{1}} x_{2}^{e_{2}}$ where $x_{0}^{e_{0}}$ is a first stack, $x_{1}^{e_{1}}$ is a lone stack, and $x_{2}^{e_{2}}$ is a last stack;

(iii) if $m \geq 3$, then $U=x_{0}^{e_{0}} x_{1}^{e_{1}} \cdots x_{m-1}^{e_{m-1}} x_{m}^{e_{m}}$, where $x_{0}^{e_{0}}$ and $x_{1}^{e_{1}}$ are first stacks and $x_{m-1}^{e_{m-1}}$ and $x_{m}^{e_{m}}$ are last stacks.

Lemma 4.5. Let $U$ be any word. Then there exists some word $\bar{U}$ in canonical form such that the identities (0) imply the identity $U \approx \bar{U}$.

Proof. By Lemmas 4.1 and 4.2, it suffices to convert the word $U$, using the identities (4.1) and (4.2), into a word in canonical form. It is clear that the word $U$ can be written in the form (4.5) that satisfies (CF1) with each $U_{i}$ being a product of interior stacks of $U$. Then the letters of $U_{i}$ can be permutated within $U_{i}$ by the identities (4.1d) in any manner. In particular, any occurrence of $x_{i-1}$ in $U_{i}$ can be moved to the left and combined with the $x_{i-1}$-stack that immediately precedes $U_{i}$, and any occurrence of $x_{i}$ in $U_{i}$ can be moved to the right and combined with the $x_{i}$-stack that immediately follows $U_{i}$, that is,

$$
U=\cdots x_{i-1}^{e_{i-1}} U_{i} x_{i}^{e_{i}} \cdots \stackrel{(4.1 \mathrm{~d})}{\approx} \cdots x_{i-1}^{p+e_{i-1}} U_{i}^{\prime} x_{i}^{q+e_{i}} \cdots
$$

where $p=\operatorname{occ}\left(x_{i-1}, U_{i}\right), q=\operatorname{occ}\left(x_{i}, U_{i}\right)$, and $U_{i}^{\prime}$ is obtained by removing all occurrences of $x_{i-1}$ and $x_{i}$ from $U_{i}$. Therefore generality is not lost by assuming that $x_{i-1}, x_{i} \notin \operatorname{con}\left(U_{i}\right)$ to begin with.

Suppose that $x^{r}$ and $y^{q}$ are adjacent entries in ext $(U)$ that violate (CF2), that is,

$$
\operatorname{ext}(U)=\left(\ldots, x^{p}, \ldots, y^{q}, x^{r}, \ldots, y^{s}, \ldots\right)
$$


where $x \neq y$ and $q$ and $r$ are even. Then

$$
U=\underbrace{\cdots x^{p} \cdots U_{i-1}}_{H} \cdot y^{q} U_{i} x^{r} \cdot \underbrace{U_{i+1} \cdots y^{s} \cdots}_{K}
$$

with $x_{i-1}^{e_{i-1}}=y^{q}$ and $x_{i}^{e_{i}}=x^{r}$. The factors $U_{i-1}, U_{i}$, and $U_{i+1}$ are products of interior stacks of $U$. In particular, $\operatorname{con}\left(U_{i}\right) \subseteq \operatorname{con}(H) \cap \operatorname{con}(K)$. Hence

$$
U \stackrel{(4.2)}{\approx} \underbrace{\cdots x^{p} \cdots U_{i-1} \cdot U_{i} x^{r} U_{i}^{11} y^{q} U_{i} \cdot U_{i+1} \cdots y^{s} \cdots}_{U\{1\}},
$$

that is, the word $U$ is converted by the identities (4.2) into the word $U^{\{1\}}$, where $\operatorname{ext}\left(U^{\{1\}}\right)$ is obtained from $\operatorname{ext}(U)$ by interchanging the entries $y^{q}$ and $x^{r}$. Note that the entries $x^{r}$ and $y^{q}$ in $\operatorname{ext}\left(U^{\{1\}}\right)$ no longer violate (CF2). Further, it is clear that $\operatorname{sep}\left(U^{\{1\}}\right)<\operatorname{sep}(U)$ or, more precisely, $\operatorname{sep}\left(U^{\{1\}}\right)=\operatorname{sep}(U)-2$.

For any $k>1$, if two adjacent entries in ext $\left(U^{\{k\}}\right)$ violate $(\mathrm{CF} 2)$, then following the interchanging procedure described in the previous paragraph, the identities (4.2) can be used to convert $U^{\{k\}}$ into a word $U^{\{k+1\}}$, where $\operatorname{ext}\left(U^{\{k+1\}}\right)$ is obtained from $\operatorname{ext}\left(U^{\{k\}}\right)$ by interchanging two adjacent entries and $\operatorname{sep}\left(U^{\{k+1\}}\right)=\operatorname{sep}\left(U^{\{k\}}\right)-2$. Since this interchanging procedure decreases the separation degree of a word by two, it can only be repeated on $U$ at most $t \leq\lfloor\operatorname{sep}(U) / 2\rfloor$ times. The word $U^{\{t\}}$ then satisfies (CF2). Therefore generality is not lost by assuming that the word $U$ satisfies (CF1) and (CF2) to begin with.

Since the factor $U_{i}$ of $U$ is a product of interior stacks of $U$, its letters can be ordered alphabetically by the identities $(4.1 \mathrm{~d})$, resulting in a word of the form $U_{i}^{\prime}=y_{1}^{f_{1}} y_{2}^{f_{2}} \cdots y_{r}^{f_{r}}$. If $y_{j}^{2}$ is a factor of $U_{i}^{\prime}$, then the identities (4.1b) can be used to gather $y_{j}^{2}$ with the last $y_{j}$-stack of $U$. This can be repeated until no letter occurs more than once in $U_{i}^{\prime}$. Hence (CF3) is satisfied.

Suppose that $x_{i-1}=x_{i}$. Then $U_{i} \neq \emptyset$ because $x_{i-1}^{e_{i-1}}$ and $x_{i}^{e_{i}}$ are stacks of $U$. Further, if $e_{i-1} \geq 2$, then

$$
\begin{aligned}
& U=\cdots x_{i-1}^{e_{i-1}} U_{i} x_{i}^{e_{i}} \cdots=\cdots x_{i}^{e_{i-1}} U_{i} x_{i}^{e_{i}} \cdots \\
& \stackrel{(4.1 \mathrm{~d})}{\approx} \cdots x_{i} U_{i} x_{i}^{e_{i}+e_{i-1}-1} \cdots \text { since } U_{i} \text { is a product of interior stacks of } U \\
& \quad=\cdots x_{i-1} U_{i} x_{i}^{e_{i}+e_{i-1}-1} \cdots,
\end{aligned}
$$

that is, the identities (4.1d) can be used to reduce the exponent of the stack $x_{i-1}^{e_{i-1}}$ to 1 . Hence (CF4) is satisfied. Finally, it is easily shown that (CF5a)-(CF5d) are satisfied by applying the identities (4.1a) and (4.1b).

\section{Proof of Theorem 1.1}

By Corollary 3.2, the variety $\mathbf{M}_{4}$ satisfies the identities (0). Therefore to show that the variety $\mathbf{M}_{4}$ is defined by the identities (0), it suffices to show 
that any identity $U \approx V$ satisfied by $\mathbf{M}_{4}$ is implied by the identities (0). Note that $\operatorname{con}(U)=\operatorname{con}(V)$ by Lemma 2.1 .

First suppose that $\operatorname{con}(U)=\operatorname{con}(V)=\{x\}$. If the identity $U \approx V$ is trivial, then it is clearly implied by the identities (0). Therefore assume that $U \neq V$, whence by Lemma 2.1, there exist some $p, q \geq 0$ such that $U=x^{3+p}$ and $V=x^{3+q}$. Further, it follows from Lemma 2.2 that $p \equiv q(\bmod 12)$. Hence the identity $U \approx V$ is implied by the identity $x^{15} \approx x^{3}$ from $(0)$.

Therefore it remains to consider the case in which $|\operatorname{con}(U)|,|\operatorname{con}(V)| \geq 2$. Then by Lemma 4.5, the words $U$ and $V$ can be assumed to be in canonical form, say

$$
U=x_{0}^{e_{0}} \prod_{i=1}^{m}\left(U_{i} x_{i}^{e_{i}}\right) \quad \text { and } \quad V=y_{0}^{f_{0}} \prod_{i=1}^{n}\left(V_{i} y_{i}^{f_{i}}\right)
$$

where $m, n \geq 1$. The results in the remainder of this section gradually prove that $U=V$. The proof of Theorem 1.1 is then complete.

Lemma 5.1. Suppose that $\left(x_{0}, x_{1}, \ldots, x_{k}\right)=\left(y_{0}, y_{1}, \ldots, y_{k}\right)$. Then the exterior stack $x_{k}^{e_{k}}$ of $U$ is lone if and only if the exterior stack $y_{k}^{f_{k}}$ of $V$ is lone.

Proof. By symmetry, it suffices to assume that the exterior stack $x_{k}^{e_{k}}$ of $U$ is lone, and then show that the exterior stack $y_{k}^{f_{k}}$ of $V$ is also lone. Seeking a contradiction, suppose that the exterior stack $y_{k}^{f_{k}}$ of $V$ is not lone. Since the exterior stack $x_{k}^{e_{k}}$ of $U$ is lone, the letter $x_{k}$ does not appear in the list $x_{0}, x_{1}, \ldots, x_{k-1}$. Therefore the letter $y_{k}=x_{k}$ does not appear in $\left(y_{0}, y_{1}, \ldots, y_{k-1}\right)=\left(x_{0}, x_{1}, \ldots, x_{k-1}\right)$, whence $y_{k}^{f_{k}}$ is a non-lone first stack of $V$, say $y_{k}=y_{\ell}$ for some $\ell>k$. For convenience, write $x=x_{k}=y_{k}=y_{\ell}$. Then by assumption,

$$
U=\underbrace{x_{0}^{e_{0}}\left(\prod_{i=1}^{k-1}\left(U_{i} x_{i}^{e_{i}}\right)\right) U_{k}}_{A} \cdot x^{e_{k}} \cdot \underbrace{\left(\prod_{i=k+1}^{m}\left(U_{i} x_{i}^{e_{i}}\right)\right)}_{B}
$$

where $x=x_{k} \notin \operatorname{con}(A B)$, and

$$
V=\underbrace{x_{0}^{f_{0}}\left(\prod_{i=1}^{k-1}\left(V_{i} x_{i}^{f_{i}}\right)\right) V_{k}}_{A^{\prime}} \cdot x^{f_{k}} \cdot \underbrace{\left(\prod_{i=k+1}^{\ell-1}\left(V_{i} y_{i}^{f_{i}}\right)\right)}_{B^{\prime}} V_{\ell} \cdot x^{f_{\ell}} \cdot \underbrace{\left(\prod_{i=\ell+1}^{n}\left(V_{i} y_{i}^{f_{i}}\right)\right)}_{C^{\prime}}
$$

where the letter $x=y_{k}=y_{\ell}$ does not appear in the list $y_{k+1}, \ldots, y_{\ell-1}$ and $x \notin \operatorname{con}\left(A^{\prime} C^{\prime}\right)$.

First suppose that $k+1=\ell$, so that $B^{\prime}=\emptyset$. Then $V_{\ell} \neq \emptyset$ by (CF4). Choose any letter $z \in \operatorname{con}\left(V_{\ell}\right)$, so that $z \neq x$ by (CF3). Then

$$
\operatorname{occ}(z, U)=\operatorname{occ}(z, A)+\operatorname{occ}(z, B)
$$




$$
\text { and } \operatorname{occ}(z, V)=\operatorname{occ}\left(z, A^{\prime}\right)+\operatorname{occ}\left(z, V_{\ell}\right)+\operatorname{occ}\left(z, C^{\prime}\right) .
$$

Since $\operatorname{occ}(z, U) \equiv \operatorname{occ}(z, V)(\bmod 2)$ by Lemma 2.2 , it follows that

(A) $\operatorname{occ}(z, A)+\operatorname{occ}(z, B) \equiv \operatorname{occ}\left(z, A^{\prime}\right)+\operatorname{occ}\left(z, V_{\ell}\right)+\operatorname{occ}\left(z, C^{\prime}\right)(\bmod 2)$.

But Lemma 2.3 implies that

(B) $\operatorname{occ}(z, A)=\operatorname{occ}(\dot{z}, x, U) \equiv \operatorname{occ}(\dot{z}, x, V)=\operatorname{occ}\left(z, A^{\prime}\right)(\bmod 2)$,

(C) $\operatorname{occ}(z, B)=\operatorname{occ}(x, \dot{z}, U) \equiv \operatorname{occ}(x, \dot{z}, V)=\operatorname{occ}\left(z, C^{\prime}\right)(\bmod 2)$.

Therefore $\operatorname{occ}\left(z, V_{\ell}\right)=0(\bmod 2)$ by $(\mathrm{A})-(\mathrm{C})$, whence $\operatorname{occ}\left(z, V_{\ell}\right)$ is positive and even. But this contradicts (CF3), so the assumption that $k+1=\ell$ is impossible.

It thus remains to assume that $k+1<\ell$. Then $B^{\prime}$ contains the exterior stack $y_{k+1}^{f_{k+1}}$ of $V$. It is shown that each of the following collectively exhaustive cases leads to a contradiction. Hence the assumption that the stack $y_{k}^{f_{k}}$ is non-lone is impossible.

CASE 1: $y_{k+1}^{f_{k+1}}$ is a last non-lone stack of $V$. Then

(D) $y_{k+1}$ is precisely one of $x_{0}, x_{1}, \ldots, x_{k-1}$,

(E) $\operatorname{occ}\left(y_{k+1}, C^{\prime}\right)=0$,

and since $y_{k+1} \notin \operatorname{con}\left(V_{k+1}\right)$ by (CF3),

(F) $\operatorname{occ}\left(y_{k+1}, B^{\prime}\right)=f_{k+1}$.

By Lemma 2.3(ii),

$$
\operatorname{fin}(U)=\operatorname{fin}(V)=\cdots y_{k+1} \cdots x \cdots,
$$

so that $y_{k+1} \notin \operatorname{con}(B)$. Therefore by (D), the $y_{k+1}$-stack of $U$ is lone and occurs in $A$, whence $\operatorname{occ}\left(y_{k+1}, U\right)=\operatorname{occ}\left(y_{k+1}, A\right)$. Further,

$$
\begin{aligned}
\operatorname{occ}\left(y_{k+1}, V\right) & =\operatorname{occ}\left(y_{k+1}, A^{\prime}\right)+\operatorname{occ}\left(y_{k+1}, B^{\prime}\right)+\operatorname{occ}\left(y_{k+1}, C^{\prime}\right) \\
& =\operatorname{occ}\left(y_{k+1}, A^{\prime}\right)+f_{k+1} \quad \text { by (E) and }(\mathrm{F}) .
\end{aligned}
$$

Therefore, as occ $\left(y_{k+1}, U\right) \equiv \operatorname{occ}\left(y_{k+1}, V\right)(\bmod 2)$ by Lemma 2.2,

(G) $\operatorname{occ}\left(y_{k+1}, A\right) \equiv \operatorname{occ}\left(y_{k+1}, A^{\prime}\right)+f_{k+1}(\bmod 2)$.

But Lemma 2.3(iii) implies that

(H) $\operatorname{occ}\left(y_{k+1}, A\right)=\operatorname{occ}\left(\dot{y}_{k+1}, x, U\right) \equiv \operatorname{occ}\left(\dot{y}_{k+1}, x, V\right)=\operatorname{occ}\left(y_{k+1}, A^{\prime}\right)$ $(\bmod 2)$.

Thus $f_{k+1} \equiv 0(\bmod 2)$ by $(G)$ and $(\mathrm{H})$, whence $f_{k+1}=2 p$ for some $p \geq 1$. Now clearly $\operatorname{occ}(x, U)=\operatorname{occ}\left(y_{k+1}, \dot{x}, U\right)$. On the other hand, $x \notin \operatorname{con}\left(V_{k+1}\right)$ by $(\mathrm{CF} 3)$, so that $\operatorname{occ}(x, V)=f_{k}+\operatorname{occ}\left(y_{k+1}, \dot{x}, V\right)$. Hence, by Lemma 2.2 ,

$$
\operatorname{occ}\left(y_{k+1}, \dot{x}, U\right) \equiv f_{k}+\operatorname{occ}\left(y_{k+1}, \dot{x}, V\right) \quad(\bmod 2) \text {. }
$$

It then follows from Lemma $2.3\left(\right.$ iv) that $f_{k} \equiv 0(\bmod 2)$, whence $f_{k}=2$ by (CF5)(b). Therefore the stacks $x^{f_{k}}=x^{2}$ and $y_{k+1}^{f_{k+1}}=y_{k+1}^{2 p}$ of $V$ violate (CF2), contradicting the assumption that the word $V$ is in canonical form. 
CASE 2: $y_{k+1}^{f_{k+1}}$ is a first stack of $V$. Then by Lemma 2.3(i),

$$
\operatorname{ini}(U)=\operatorname{ini}(V)=\cdots x \cdots y_{k+1} \cdots,
$$

so that every $y_{k+1}$-stack of $U$ occurs in $B$, that is,

(I) $\operatorname{occ}\left(y_{k+1}, U\right)=\operatorname{occ}\left(y_{k+1}, B\right)$.

Hence by Lemma 2.3(ii),

$$
\operatorname{fin}(V)=\operatorname{fin}(U)=\cdots x \cdots y_{k+1} \cdots .
$$

It follows that the factor $C^{\prime}$ of $V$ must contain the letter $y_{k+1}$. Therefore

( $\mathrm{J}$ ) the stack $y_{k+1}^{f_{k+1}}$ of $V$ is a non-lone first stack, while the last $y_{k+1}$-stack of $V$ occurs in $C^{\prime}$.

SubCASE 2.1: $k+1=\ell-1$. Then

$$
V=\underbrace{x_{0}^{f_{0}}\left(\prod_{i=1}^{k-1}\left(V_{i} x_{i}^{f_{i}}\right)\right) V_{k}}_{A^{\prime}} \cdot x^{f_{k}} \cdot \underbrace{V_{k+1} y_{k+1}^{f_{k+1}}}_{B^{\prime}} V_{\ell} \cdot x^{f_{\ell}} \cdot \underbrace{\left(\prod_{i=\ell+1}^{n}\left(V_{i} y_{i}^{f_{i}}\right)\right)}_{C^{\prime}} .
$$

Since $y_{k+1}=y_{\ell-1} \notin \operatorname{con}\left(V_{\ell}\right)$ by $(\mathrm{CF} 3)$,

(K) $\operatorname{occ}\left(y_{k+1}, V\right)=f_{k+1}+\operatorname{occ}\left(y_{k+1}, C^{\prime}\right)$.

Further, since $\operatorname{occ}\left(y_{k+1}, U\right) \equiv \operatorname{occ}\left(y_{k+1}, V\right)(\bmod 2)$, by Lemma 2.2, it follows from (I) and (K) that

(L) $\operatorname{occ}\left(y_{k+1}, B\right) \equiv f_{k+1}+\operatorname{occ}\left(y_{k+1}, C^{\prime}\right)(\bmod 2)$.

But Lemma 2.3(iv) implies that

$$
\operatorname{occ}\left(y_{k+1}, B\right)=\operatorname{occ}\left(x, \dot{y}_{k+1}, U\right) \equiv \operatorname{occ}\left(x, \dot{y}_{k+1}, V\right)=\operatorname{occ}\left(y_{k+1}, C^{\prime}\right) \quad(\bmod 2),
$$

whence $f_{k+1} \equiv 0(\bmod 2)$ by $(\mathrm{L})$. Thus $f_{k+1}=2 p$ for some $p \geq 1$. Now

(M) $\operatorname{occ}(x, U)=\operatorname{occ}\left(\dot{x}, y_{k+1}, U\right)$

by (I). On the other hand, $x \notin \operatorname{con}\left(V_{k+1} V_{\ell}\right)$ by (CF3), so that

(N) $\operatorname{occ}(x, V)=\operatorname{occ}\left(\dot{x}, y_{k+1}, V\right)+f_{\ell}$.

Since $\operatorname{occ}(x, U) \equiv \operatorname{occ}(x, V)(\bmod 2)$, by Lemma 2.2 , it follows from (M) and $(\mathrm{N})$ that

$$
\operatorname{occ}\left(\dot{x}, y_{k+1}, U\right) \equiv \operatorname{occ}\left(\dot{x}, y_{k+1}, V\right)+f_{\ell}(\bmod 2)
$$

whence $f_{\ell} \equiv 0(\bmod 2)$ by Lemma 2.3 (iii). Thus $f_{\ell}=2 q$ for some $q \geq 1$. Consequently, the stacks $y_{k+1}^{f_{k+1}}=y_{k+1}^{2 p}$ and $x^{f_{\ell}}=x^{2 q}$ of $V$ violate (CF2), contradicting the assumption that the word $V$ is in canonical form. 
SubCASE 2.2: $k+1<\ell-1$. Then

$$
\begin{aligned}
& V=\overbrace{x_{0}^{f_{0}}\left(\prod_{i=1}^{k-1}\left(V_{i} x_{i}^{f_{i}}\right)\right) V_{k}}^{A^{\prime}} \cdot x^{f_{k}} \cdot \overbrace{V_{k+1} y_{k+1}^{f_{k+1}} \cdot V_{k+2} y_{k+2}^{f_{k+2}} \cdots}^{B^{\prime}} V_{\ell} \cdot x^{f_{\ell}} . \\
& \underbrace{\left(\prod_{i=\ell+1}^{n}\left(V_{i} y_{i}^{f_{i}}\right)\right)}_{C^{\prime}}
\end{aligned}
$$

and $y_{k+1} \neq y_{k+2}$ by ( $\left.\mathrm{J}\right)$. Seeking a contradiction, suppose that

(†) $y_{k+2}^{f_{k+2}}$ is a non-lone last stack of $V$.

Then the first $y_{k+2}$-stack of $V$ occurs in $A^{\prime}$, so that

(0) $y_{k+2}$ coincides with precisely one of $x_{0}, x_{1}, \ldots, x_{k-1}$.

Since $y_{k+2} \notin \operatorname{con}\left(V_{k+2}\right)$ by (CF3), it follows from ( $\dagger$ ) that

(P) $\operatorname{occ}\left(y_{k+2}, B^{\prime}\right)=\operatorname{occ}\left(y_{k+2}, V_{k+1}\right)+f_{k+2}$.

It is clear that $\operatorname{occ}\left(y_{k+2}, C^{\prime}\right)=0$ by $(\dagger)$. Therefore

$$
\begin{aligned}
\operatorname{occ}\left(y_{k+2}, V\right) & =\operatorname{occ}\left(y_{k+2}, A^{\prime}\right)+\operatorname{occ}\left(y_{k+2}, B^{\prime}\right)+\operatorname{occ}\left(y_{k+2}, C^{\prime}\right) \\
& =\operatorname{occ}\left(y_{k+2}, A^{\prime}\right)+\operatorname{occ}\left(y_{k+2}, V_{k+1}\right)+f_{k+2}+0
\end{aligned}
$$

by (P), whence

(Q) $\operatorname{occ}\left(y_{k+2}, V\right)=\operatorname{occ}\left(\dot{y}_{k+2}, y_{k+1}, V\right)+f_{k+2}$.

Now

$$
\begin{aligned}
\operatorname{fin}(U) & =\operatorname{fin}(V) & & \text { by Lemma 2.3(ii) } \\
& =\cdots y_{k+2} \cdots x \cdots y_{k+1} \cdots & & \text { by ( } \mathrm{J} \text { ) and (0). }
\end{aligned}
$$

Therefore, as $x^{e_{k}}$ is a lone stack of $U$,

(R) every occurrence of $y_{k+2}$ in $U$ precedes $x^{e_{k}}$ while every occurrence of $y_{k+1}$ in $U$ follows $x^{e_{k}}$.

Hence

(S) $\operatorname{occ}\left(y_{k+2}, U\right)=\operatorname{occ}\left(\dot{y}_{k+2}, y_{k+1}, U\right)$.

Since occ $\left(y_{k+2}, U\right) \equiv \operatorname{occ}\left(y_{k+2}, V\right)(\bmod 2)$ by Lemma 2.2 , it follows from (Q) and $(\mathrm{S})$ that

$$
\operatorname{occ}\left(\dot{y}_{k+2}, y_{k+1}, U\right) \equiv \operatorname{occ}\left(\dot{y}_{k+2}, y_{k+1}, V\right)+f_{k+2} \quad(\bmod 2) .
$$

It then follows from Lemma $2.3\left(\right.$ iii) that $f_{k+2} \equiv 0(\bmod 2)$, whence $f_{k+2}=2 q$ for some $q \geq 1$.

It is clear that $(R)$ implies that

( $\mathrm{T}) \operatorname{occ}\left(y_{k+1}, U\right)=\operatorname{occ}\left(y_{k+2}, \dot{y}_{k+1}, U\right)$.

Further, $y_{k+1} \notin \operatorname{con}\left(V_{k+2}\right)$ by $(\mathrm{CF} 3)$, so that $(\mathrm{J})$ and $(\dagger)$ imply that

(U) $\operatorname{occ}\left(y_{k+1}, V\right)=f_{k+1}+\operatorname{occ}\left(y_{k+2}, \dot{y}_{k+1}, V\right)$. 
Therefore Lemma 2.2, (T), and (U) imply that

$$
\operatorname{occ}\left(y_{k+2}, \dot{y}_{k+1}, U\right) \equiv f_{k+1}+\operatorname{occ}\left(y_{k+2}, \dot{y}_{k+1}, V\right) \quad(\bmod 2) \text {. }
$$

It then follows from Lemma $2.3($ iv $)$ that $f_{k+1} \equiv 0(\bmod 2)$, whence $f_{k+1}=2$ by (CF5)(b). Now the stacks $y_{k+1}^{f_{k+1}}=y_{k+1}^{2}$ and $x^{f_{k+1}}=x^{2 q}$ of $V$ violate (CF2), contradicting the assumption that the word $V$ is in canonical form. The assumption $(\dagger)$ thus cannot hold, whence $y_{k+2}^{f_{k+2}}$ is a first stack of $V$. Note that if all $y_{k+2}$-stacks of $V$ occur in $B^{\prime}$, then

$$
\operatorname{ini}(V)=\cdots x \cdots y_{k+2} \cdots \quad \text { and } \quad \operatorname{fin}(V)=\cdots y_{k+2} \cdots x \cdots .
$$

But since ini $(U)=\operatorname{ini}(V)$ and $\operatorname{fin}(U)=\operatorname{fin}(V)$ by Lemma 2.3, every occurrence of $y_{k+2}$ in $U$ is sandwiched by two occurrences of $x$; this is impossible because $x^{e_{k}}$ is a lone stack of $U$. Consequently,

(V) $y_{k+2}^{f_{k+2}}$ is a first stack of $V$ and the last $y_{k+2}$-stack of $V$ occurs in $C^{\prime}$.

By repeating the arguments that deduced (V), it can be shown that for each $i \in\{k+2, k+3, \ldots, \ell-1\}$, the exterior stack $y_{i}^{f_{i}}$ of $V$ is a first stack, while the last $y_{i}$-stack of $V$ occurs in $C^{\prime}$. By repeating the same argument on the stacks $y_{\ell-1}^{f_{\ell-1}}$ and $x^{f_{\ell}}=y_{\ell}^{f_{\ell}}$, both $f_{\ell-1}$ and $f_{\ell}$ can be shown to be positive even integers; these stacks then violate (CF2).

Lemma 5.2. Suppose that $\left(x_{0}, x_{1}, \ldots, x_{k-1}\right)=\left(y_{0}, y_{1}, \ldots, y_{k-1}\right)$. Then $x_{k}=y_{k}$.

Proof. It is notationally less cumbersome to write $x=x_{k}$ and $y=y_{k}$. Suppose that $x \neq y$. Then

$$
U=\underbrace{x_{0}^{e_{0}}\left(\prod_{i=1}^{k-1}\left(U_{i} x_{i}^{e_{i}}\right)\right) U_{k}}_{A} \cdot x^{e_{k}} \cdot \underbrace{\left(\prod_{i=k+1}^{m}\left(U_{i} x_{i}^{e_{i}}\right)\right)}_{B}
$$

and

$$
V=\underbrace{x_{0}^{f_{0}}\left(\prod_{i=1}^{k-1}\left(V_{i} x_{i}^{f_{i}}\right)\right) V_{k}}_{A^{\prime}} \cdot y^{f_{k}} \cdot \underbrace{\left(\prod_{i=k+1}^{n}\left(V_{i} y_{i}^{f_{i}}\right)\right)}_{B^{\prime}} .
$$

It is shown that each of the following collectively exhaustive cases leads to a contradiction. Hence the assumption that $x \neq y$ is impossible.

CASE 1: $x^{e_{k}}$ is a first stack of $U$ and $y^{f_{k}}$ is a first stack of $V$. Then $x \notin \operatorname{con}(A)$ and $y \notin \operatorname{con}\left(A^{\prime}\right)$, so that

(A) $\operatorname{ini}(U)=\operatorname{ini}(A) x \cdots$ and $\operatorname{ini}(V)=\operatorname{ini}\left(A^{\prime}\right) y \cdots$.

$\operatorname{But} \operatorname{ini}(U)=\operatorname{ini}(V)$ by Lemma $2.3(\mathrm{i})$ and $\operatorname{ini}(A)=\operatorname{ini}\left(x_{0} x_{1} \cdots x_{k-1}\right)=\operatorname{ini}\left(A^{\prime}\right)$. Therefore the contradiction $x=y$ follows from (A).

CASE 2: $x^{e_{k}}$ is a last stack of $U$ and $y^{f_{k}}$ is a last stack of $V$. By Case 1, it is not possible for $x^{e_{k}}$ to be lone in $U$ and $y^{f_{k}}$ to be lone in $V$ simultaneously. 
Therefore by symmetry, it suffices to assume that the stack $x^{e_{k}}$ is not lone in $U$, whence

(B) $x$ appears precisely once in the list $x_{0}, x_{1}, \ldots, x_{k-1}$, say $x=x_{j}$ for some $j \in\{0,1, \ldots, k-1\}$. Hence

$$
U=\underbrace{x_{0}^{e_{0}}\left(\prod_{i=1}^{j-1}\left(U_{i} x_{i}^{e_{i}}\right)\right) U_{j}}_{A_{1}} \cdot x^{e_{j}} \cdot \underbrace{\left(\prod_{i=j+1}^{k-1}\left(U_{i} x_{i}^{e_{i}}\right)\right) U_{k}}_{A_{2}} \cdot x^{e_{k}} \cdot \underbrace{\left(\prod_{i=k+1}^{m}\left(U_{i} x_{i}^{e_{i}}\right)\right)}_{B}
$$

and

$$
V=\underbrace{x_{0}^{f_{0}}\left(\prod_{i=1}^{j-1}\left(V_{i} x_{i}^{f_{i}}\right)\right) V_{j}}_{A_{1}^{\prime}} \cdot x^{f_{j}} \cdot \underbrace{\left(\prod_{i=j+1}^{k-1}\left(V_{i} x_{i}^{f_{i}}\right)\right) V_{k}}_{A_{2}^{\prime}} \cdot y^{f_{k}} \cdot \underbrace{\left(\prod_{i=k+1}^{n}\left(V_{i} y_{i}^{f_{i}}\right)\right)}_{B^{\prime}}
$$

where $A=A_{1} x^{e_{j}} A_{2}$ and $A^{\prime}=A_{1}^{\prime} x^{f_{j}} A_{2}^{\prime}$. By Lemma 5.1, the stack $x^{f_{j}}$ of $V$ cannot be lone. Thus by (B), the last $x$-stack of $V$ occurs in $B^{\prime}$, so that fin $(V)=\cdots y \cdots x \cdots$. Therefore $\operatorname{fin}(U)=\cdots y \cdots x \cdots$ by Lemma 2.3(ii), whence the last $y$-stack of $U$ occurs in $A_{1}$ or $A_{2}$. If this last $y$-stack of $U$ is not lone, then precisely two of $x_{0}, \ldots, x_{j-1}, x_{j+1}, \ldots, x_{k-1}$ coincide with $y$, whence there are three exterior $y$-stacks in $V$, which is clearly impossible. Therefore the $y$-stack in $U$ is lone; in this case, Lemma 5.1 is violated.

CASE 3: $x^{e_{k}}$ is a first stack of $U$ and $y^{f_{k}}$ is a last stack of $V$. By Cases 1 and 2, neither the stack $x^{e_{k}}$ of $U$ nor the stack $y^{f_{k}}$ of $V$ can be lone, thus

(C) $x \notin \operatorname{con}(A)$ and $x \in \operatorname{con}(B)$;

(D) the first $y$-stack of $V$ occurs in $A^{\prime}$ (so that $y$ appears precisely once in the list $\left.x_{0}, x_{1}, \ldots, x_{k-1}\right)$ and $y \notin \operatorname{con}\left(B^{\prime}\right)$.

Since

$$
\begin{aligned}
\operatorname{ini}(V) & =\operatorname{ini}(U) & & \text { by Lemma } 2.3(\mathrm{i}) \\
& =\cdots y \cdots x \cdots & & \text { by (C) and (D), }
\end{aligned}
$$

the first $x$-stack of $V$ follows the first $y$-stack of $V$. If $x \in \operatorname{con}\left(A^{\prime}\right)$, so that the first $x$-stack of $V$ occurs in $A^{\prime}$, then $x$ is precisely one of $x_{0}, x_{1}, \ldots, x_{k-1}$, violating (C). Therefore

(E) $x \notin \operatorname{con}\left(A^{\prime}\right)$ and $x \in \operatorname{con}\left(B^{\prime}\right)$.

It follows from (D) and (E) that $\operatorname{fin}(V)=\cdots y \cdots x \cdots$. But $\operatorname{fin}(U)=\operatorname{fin}(V)$ by Lemma 2.3(ii), so that $\operatorname{fin}(U)=\cdots y \cdots x \cdots$. Therefore

(F) the last $y$-stack of $U$ precedes the last $x$-stack of $U$, say $y=x_{\ell}$ for some $\ell>k$. 
Hence

$$
U=\underbrace{x_{0}^{e_{0}}\left(\prod_{i=1}^{k-1}\left(U_{i} x_{i}^{e_{i}}\right)\right) U_{k}}_{A} \cdot x^{e_{k}} \cdot \underbrace{\left(\prod_{i=k+1}^{\ell-1}\left(U_{i} x_{i}^{e_{i}}\right)\right) U_{\ell}}_{B_{1}} \cdot y^{e_{\ell}} \cdot \underbrace{\left(\prod_{i=\ell+1}^{m}\left(U_{i} x_{i}^{e_{i}}\right)\right)}_{B_{2}}
$$

and

$$
V=\underbrace{x_{0}^{f_{0}}\left(\prod_{i=1}^{k-1}\left(V_{i} x_{i}^{f_{i}}\right)\right) V_{k}}_{A^{\prime}} \cdot y^{f_{k}} \cdot \underbrace{\left(\prod_{i=k+1}^{n}\left(V_{i} y_{i}^{f_{i}}\right)\right)}_{B^{\prime}}
$$

where $B=B_{1} y^{e_{\ell}} B_{2}$ with the last $x$-stack of $U$ occurring in $B_{2}$. Since $x^{e_{k}}$ is the first $x$-stack of $U$, none of the exterior stacks in $B_{1}$ can be an $x$-stack, whence

(G) $x$ does not appear in the list $x_{k+1}, \ldots, x_{\ell-1}$.

SubCASE 3.1: $k<\ell-1$. Then $x_{\ell-1}^{e_{\ell-1}}$ is an exterior stack of $U$ that occurs in $B_{1}$ and $x_{\ell-1} \neq x$ by (G). Further, $x_{\ell-1} \neq y$ by (D). Suppose that $x_{\ell-1}^{e_{\ell-1}}$ is a last stack of $U$, so that $\operatorname{fin}(U)=\cdots x_{\ell-1} \cdots y \cdots$. Then $\operatorname{fin}(V)=\cdots x_{\ell-1} \cdots y \cdots$ by Lemma 2.3(ii), whence the last $x_{\ell-1}$-stack of $V$ occurs in $A^{\prime}$, say $x_{\ell-1}=x_{q}$ for some $q \leq k-1$. If there are two exterior $x_{\ell-1}$-stacks in $V$, say $x_{\ell-1}=x_{p}$ with $p<q$, then $x_{p}^{e_{p}}, x_{q}^{e_{q}}$, and $x_{\ell-1}^{e_{\ell-1}}$ are three distinct exterior $x_{\ell-1}$-stacks of $U$, which is impossible. Hence the $x_{\ell-1}$-stack $x_{q}^{f_{q}}$ of $V$ is lone. But now the $x_{\ell-1}$-stack $x_{q}^{e_{q}}$ of $U$ is not lone, and this violates Lemma 5.1. Therefore $x_{\ell-1}^{e_{\ell-1}}$ is a non-lone first stack of $U$, whence

(H) $x_{\ell-1}$ does not appear in the list $x_{0}, x_{1}, \ldots, x_{k-1}$,

(I) the last $x_{\ell-1}$-stack of $U$ occurs in $B_{2}$.

Now Lemma 2.3(i) implies that $\operatorname{ini}(V)=\operatorname{ini}(U)=\cdots x \cdots x_{\ell-1} \cdots$, whence the first $x_{\ell-1}$-stack of $V$ follows the first $x$-stack of $V$. It then follows from (I) that

(J) the first $x_{\ell-1}$-stack of $V$ occurs in $B^{\prime}$,

whence $\operatorname{occ}(y, V)=\operatorname{occ}\left(\dot{y}, x_{\ell-1}, V\right)$. Further, (D) implies that the first $y$ stack of $U$ occurs in $A$, so (F) implies that $\operatorname{occ}(y, U)=\operatorname{occ}\left(\dot{y}, x_{\ell-1}, U\right)+e_{\ell}$. Therefore

$$
\operatorname{occ}\left(\dot{y}, x_{\ell-1}, V\right) \equiv \operatorname{occ}\left(\dot{y}, x_{\ell-1}, U\right)+e_{\ell} \quad(\bmod 2)
$$

by Lemma 2.2. But

$$
\operatorname{occ}\left(\dot{y}, x_{\ell-1}, V\right) \equiv \operatorname{occ}\left(\dot{y}, x_{\ell-1}, U\right) \quad(\bmod 2)
$$

by Lemma 2.3(iii), so that $e_{\ell}$ is even. On the other hand,

$$
\begin{aligned}
& \operatorname{occ}\left(x_{\ell-1}, U\right)=e_{\ell-1}+\operatorname{occ}\left(y, \dot{x}_{\ell-1}, U\right) \quad \text { by (F) and (I) } \\
& \text { and } \operatorname{occ}\left(x_{\ell-1}, V\right)=\operatorname{occ}\left(y, \dot{x}_{\ell-1}, V\right) \quad \text { by }(\mathrm{J}) \text {. }
\end{aligned}
$$


Therefore

$$
e_{\ell-1}+\operatorname{occ}\left(y, \dot{x}_{\ell-1}, U\right) \equiv \operatorname{occ}\left(y, \dot{x}_{\ell-1}, V\right) \quad(\bmod 2)
$$

by Lemma 2.2. But

$$
\operatorname{occ}\left(y, \dot{x}_{\ell-1}, U\right) \equiv \operatorname{occ}\left(y, \dot{x}_{\ell-1}, V\right) \quad(\bmod 2)
$$

by Lemma 2.3(iv), so that $e_{\ell-1}$ is even. Now since $y \in \operatorname{con}(A)$ by (D) and $x_{\ell-1} \in \operatorname{con}\left(B_{2}\right)$ by (I), the stacks $x_{\ell-1}^{e_{\ell-1}}$ and $y^{e_{\ell}}$ of $U$ violate (CF2), contradicting the assumption that the word $U$ is in canonical form.

SubCASE 3.2: $k=\ell-1$. Then

$$
U=\underbrace{x_{0}^{e_{0}}\left(\prod_{i=1}^{k-1}\left(U_{i} x_{i}^{e_{i}}\right)\right) U_{k}}_{A} \cdot x^{e_{k}} \cdot U_{\ell} \cdot y^{e_{\ell}} \cdot \underbrace{\left(\prod_{i=\ell+1}^{m}\left(U_{i} x_{i}^{e_{i}}\right)\right)}_{B_{2}}
$$

and

$$
V=\underbrace{x_{0}^{f_{0}}\left(\prod_{i=1}^{k-1}\left(V_{i} x_{i}^{f_{i}}\right)\right) V_{k}}_{A^{\prime}} \cdot y^{f_{k}} \cdot \underbrace{\left(\prod_{i=k+1}^{n}\left(V_{i} y_{i}^{f_{i}}\right)\right)}_{B^{\prime}}
$$

where $B=U_{\ell} y^{e_{\ell}} B_{2}$. By (CF3),

(K) $x, y \notin \operatorname{con}\left(U_{\ell}\right)$.

Then (C) and (K) imply that $\operatorname{occ}(x, U)=e_{k}+\operatorname{occ}(y, \dot{x}, U)$, and (D) and (E) imply that $\operatorname{occ}(x, V)=\operatorname{occ}(y, \dot{x}, V)$. Therefore

$$
e_{k}+\operatorname{occ}(y, \dot{x}, U) \equiv \operatorname{occ}(y, \dot{x}, V) \quad(\bmod 2)
$$

by Lemma 2.2. But

$$
\operatorname{occ}(y, \dot{x}, V) \equiv \operatorname{occ}(y, \dot{x}, U) \quad(\bmod 2)
$$

by Lemma 2.3(iv), so that $e_{\ell}$ is even. On the other hand,

$$
\begin{aligned}
& \operatorname{occ}(y, U)=\operatorname{occ}(\dot{y}, x, U)+e_{\ell} \quad \text { by }(\mathrm{K}) \\
& \text { and } \operatorname{occ}(y, V)=\operatorname{occ}(\dot{y}, x, V) \quad \text { by (C). }
\end{aligned}
$$

Therefore

$$
\operatorname{occ}(\dot{y}, x, U)+e_{\ell} \equiv \operatorname{occ}(\dot{y}, x, V) \quad(\bmod 2)
$$

by Lemma 2.2. But

$$
\operatorname{occ}(\dot{y}, x, U) \equiv \operatorname{occ}(\dot{y}, x, V) \quad(\bmod 2)
$$

by Lemma 2.3(iii), so that $e_{\ell}$ is even. Now since $y \in \operatorname{con}(A)$ by (D) and $x \in \operatorname{con}\left(B_{2}\right)$ by $(\mathrm{C})$ and $(\mathrm{K})$, the stacks $x^{e_{k}}$ and $y^{e_{\ell}}$ of $U$ violate (CF2), contradicting the assumption that the word $U$ is in canonical form. 
Lemma 5.3. $m=n$ and $x_{k}=y_{k}$ for all $k$.

Proof. Suppose that $m<n$. Since $\operatorname{ini}(U)=\operatorname{ini}(V)$ by Lemma 2.3(i), it follows that $x_{0}=y_{0}$. Thus $\left(x_{0}, x_{1}, \ldots, x_{m}\right)=\left(y_{0}, y_{1}, \ldots, y_{m}\right)$ by Lemma 5.2 , whence

$$
U=x_{0}^{e_{0}} \prod_{i=1}^{m}\left(U_{i} x_{i}^{e_{i}}\right) \quad \text { and } \quad V=x_{0}^{f_{0}}\left(\prod_{i=1}^{m}\left(V_{i} x_{i}^{f_{i}}\right)\right)\left(\prod_{i=m+1}^{n}\left(V_{i} y_{i}^{f_{i}}\right)\right) .
$$

Now $\operatorname{fin}(U)=\operatorname{fin}(V)$ by Lemma 2.3(ii), so that $x_{m}=y_{n}$. Therefore there are two $x_{m}$-stacks in $V$; the first $x_{m}$-stack is $x_{m}^{f_{m}}$ while the last $x_{m}$-stack is $y_{n}^{f_{n}}$. It follows that the letter $x_{m}$ in $V$ does not appear in the list $x_{0}, x_{1}, \ldots, x_{m-1}$, whence the $x_{m}$-stack of $U$ is lone. Lemma 5.1 is thus violated.

Therefore the assumption $m<n$ is impossible. By symmetry, it is also impossible for $m>n$. Consequently, $m=n$ and the lemma holds.

Lemma 5.4. $e_{k} \equiv f_{k}(\bmod 2)$ for all $k$.

Proof. Since $x_{0} \neq x_{1}$ and $U_{1}=V_{1}=\emptyset$, by Remark 4.4, it follows from Lemma 2.3(iii) that

$$
e_{0}=\operatorname{occ}\left(\dot{x}_{0}, x_{1}, U\right) \equiv \operatorname{occ}\left(\dot{x}_{0}, x_{1}, V\right)=f_{0} \quad(\bmod 2) .
$$

Symmetrically, $e_{m} \equiv f_{m}(\bmod 2)$ by Lemma 2.3(iv). Therefore assume that $0<k<m$, whence

$$
U=\underbrace{x_{0}^{e_{0}}\left(\prod_{i=1}^{k-1}\left(U_{i} x_{i}^{e_{i}}\right)\right)}_{A} \cdot U_{k} \cdot x_{k}^{e_{k}} \cdot U_{k+1} \cdot \underbrace{x_{k+1}^{e_{k+1}}\left(\prod_{i=k+2}^{m}\left(U_{i} x_{i}^{e_{i}}\right)\right)}_{B}
$$

and

$$
V=\underbrace{x_{0}^{f_{0}}\left(\prod_{i=1}^{k-1}\left(V_{i} x_{i}^{f_{i}}\right)\right)}_{A^{\prime}} \cdot V_{k} \cdot x_{k}^{f_{k}} \cdot V_{k+1} \cdot \underbrace{x_{k+1}^{f_{k+1}}\left(\prod_{i=k+2}^{m}\left(V_{i} x_{i}^{f_{i}}\right)\right)}_{B^{\prime}} .
$$

If $x_{k-1}=x_{k}$, then as $x_{k-1}=x_{k} \notin \operatorname{con}\left(U_{k} V_{k}\right)$ by (CF3) and $e_{k-1}=1=f_{k-1}$ by $(\mathrm{CF} 4)$, it follows from Lemma 2.2 that

$$
\begin{aligned}
1+e_{k}=e_{k-1}+e_{k}=\operatorname{occ}\left(x_{k}, U_{k}\right) & \equiv \operatorname{occ}\left(x_{k}, V_{k}\right) \\
& =f_{k-1}+f_{k}=1+f_{k} \quad(\bmod 2),
\end{aligned}
$$

whence $e_{k} \equiv f_{k}(\bmod 2)$. If $x_{k}=x_{k+1}$, then $e_{k}=1=f_{k}$ by $(\mathrm{CF} 4)$. Therefore it remains to assume that $x_{k-1} \neq x_{k} \neq x_{k+1}$. Then $x_{k} \notin \operatorname{con}\left(U_{k} U_{k+1} V_{k} V_{k+1}\right)$ by (CF3), so that

$$
\operatorname{occ}\left(x_{k}, U_{k}\right)=\operatorname{occ}\left(x_{k}, U_{k+1}\right)=\operatorname{occ}\left(x_{k}, V_{k}\right)=\operatorname{occ}\left(x_{k}, V_{k+1}\right)=0 .
$$

There are four cases to consider. 
CASE 1: $x_{k-1}^{e_{k-1}}$ and $x_{k-1}^{f_{k-1}}$ are first stacks of $U$ and $V$ respectively, while $x_{k+1}^{e_{k+1}}$ and $x_{k+1}^{f_{k+1}}$ are first stacks of $U$ and $V$ respectively. Then

$$
\begin{aligned}
\operatorname{occ}\left(\dot{x}_{k}, x_{k+1}, U\right) & =\operatorname{occ}\left(x_{k}, A\right)+\operatorname{occ}\left(x_{k}, U_{k}\right)+e_{k}+\operatorname{occ}\left(x_{k}, U_{k+1}\right) \\
& =\operatorname{occ}\left(\dot{x}_{k}, x_{k-1}, U\right)+0+e_{k}+0 .
\end{aligned}
$$

Similarly, $\operatorname{occ}\left(\dot{x}_{k}, x_{k+1}, V\right)=\operatorname{occ}\left(\dot{x}_{k}, x_{k-1}, V\right)+f_{k}$. Hence $e_{k} \equiv f_{k}(\bmod 2)$ by Lemma 2.3 (iii).

CASE 2: $x_{k-1}^{e_{k-1}}$ and $x_{k-1}^{f_{k-1}}$ are last stacks of $U$ and $V$ respectively, while $x_{k+1}^{e_{k+1}}$ and $x_{k+1}^{f_{k+1}}$ are last stacks of $U$ and $V$ respectively. Then $e_{k} \equiv f_{k}(\bmod 2)$ by an argument symmetrical to Case 1 .

CASE 3: $x_{k-1}^{e_{k-1}}$ and $x_{k-1}^{f_{k-1}}$ are first stacks of $U$ and $V$ respectively, while $x_{k+1}^{e_{k+1}}$ and $x_{k+1}^{f_{k+1}}$ are last stacks of $U$ and $V$ respectively. Then

$$
\begin{aligned}
\operatorname{occ}\left(x_{k}, U\right) & =\operatorname{occ}\left(x_{k}, A\right)+\operatorname{occ}\left(x_{k}, U_{k}\right)+e_{k}+\operatorname{occ}\left(x_{k}, U_{k+1}\right)+\operatorname{occ}\left(x_{k}, B\right) \\
& =\operatorname{occ}\left(\dot{x}_{k}, x_{k-1}, U\right)+0+e_{k}+0+\operatorname{occ}\left(x_{k+1}, \dot{x}_{k}, U\right) .
\end{aligned}
$$

Similarly, $\operatorname{occ}\left(x_{k}, V\right)=\operatorname{occ}\left(\dot{x}_{k}, x_{k-1}, V\right)+f_{k}+\operatorname{occ}\left(x_{k+1}, \dot{x}_{k}, V\right)$. It then follows from Lemmas 2.2, 2.3(iii) and 2.3(iv) that $e_{k} \equiv f_{k}(\bmod 2)$.

CASE 4: $x_{k-1}^{e_{k-1}}$ and $x_{k-1}^{f_{k-1}}$ are last stacks of $U$ and $V$ respectively, while $x_{k+1}^{e_{k+1}}$ and $x_{k+1}^{f_{k+1}}$ are first stacks of $U$ and $V$ respectively. Then

$$
\begin{aligned}
\operatorname{occ}\left(\dot{x}_{k}, x_{k+1}, U\right) & =\operatorname{occ}\left(x_{k}, A\right)+\operatorname{occ}\left(x_{k}, U_{k}\right)+e_{k}+\operatorname{occ}\left(x_{k}, U_{k+1}\right) \\
& =\operatorname{occ}\left(x_{k}, A\right)+0+e_{k}+0 \\
\text { and } \operatorname{occ}\left(x_{k-1}, \dot{x}_{k}, U\right) & =\operatorname{occ}\left(x_{k}, U_{k}\right)+e_{k}+\operatorname{occ}\left(x_{k}, U_{k+1}\right)+\operatorname{occ}\left(x_{k}, B\right) \\
& =0+e_{k}+0+\operatorname{occ}\left(x_{k}, B\right),
\end{aligned}
$$

so that

$$
\begin{aligned}
\operatorname{occ}\left(x_{k}, A\right) & =\operatorname{occ}\left(\dot{x}_{k}, x_{k+1}, U\right)-e_{k} \\
\text { and } \quad \operatorname{occ}\left(x_{k}, B\right) & =\operatorname{occ}\left(x_{k-1}, \dot{x}_{k}, U\right)-e_{k} .
\end{aligned}
$$

Hence

$$
\begin{aligned}
\operatorname{occ}\left(x_{k}, U\right) & =\operatorname{occ}\left(x_{k}, A\right)+\operatorname{occ}\left(x_{k}, U_{k}\right)+e_{k}+\operatorname{occ}\left(x_{k}, U_{k+1}\right)+\operatorname{occ}\left(x_{k}, B\right) \\
& =\operatorname{occ}\left(\dot{x}_{k}, x_{k+1}, U\right)-e_{k}+0+e_{k}+0+\operatorname{occ}\left(x_{k-1}, \dot{x}_{k}, U\right)-e_{k} \\
& =\operatorname{occ}\left(\dot{x}_{k}, x_{k+1}, U\right)+\operatorname{occ}\left(x_{k-1}, \dot{x}_{k}, U\right)-e_{k} .
\end{aligned}
$$

Similarly, $\operatorname{occ}\left(x_{k}, V\right)=\operatorname{occ}\left(\dot{x}_{k}, x_{k+1}, V\right)+\operatorname{occ}\left(x_{k-1}, \dot{x}_{k}, V\right)-f_{k}$. It then follows from Lemmas 2.2, 2.3(iii), and 2.3(iv) that $e_{k} \equiv f_{k}(\bmod 2)$.

LEMMA 5.5. $U_{k}=V_{k}$ for all $k$.

Proof. By Lemmas 5.3 and 5.4,

$$
U=x_{0}^{e_{0}} \prod_{i=1}^{m}\left(U_{i} x_{i}^{e_{i}}\right) \quad \text { and } \quad V=x_{0}^{f_{0}} \prod_{i=1}^{m}\left(V_{i} x_{i}^{f_{i}}\right)
$$


where $m \geq 1$ and

(A) $e_{i} \equiv f_{i}(\bmod 2)$ for all $i$.

It is clear that $U_{1}=\emptyset=V_{1}$ by (CF3). Suppose that

$$
\left(U_{1}, U_{2}, \ldots, U_{k-1}\right)=\left(V_{1}, V_{2}, \ldots, V_{k-1}\right)
$$

where $k \geq 1$. Then it follows from (A) that

(B) $\operatorname{occ}\left(y, x_{0}^{e_{0}} \prod_{i=1}^{k-1}\left(U_{i} x_{i}^{e_{i}}\right)\right) \equiv \operatorname{occ}\left(y, x_{0}^{f_{0}} \prod_{i=1}^{k-1}\left(V_{i} x_{i}^{f_{i}}\right)\right)(\bmod 2)$ for all $y \in \Sigma$.

The present lemma is thus established by induction once it is shown that $U_{k}=V_{k}$. Now by (CF3), the letters of $U_{k}$ and of $V_{k}$ are in strict alphabetical order, so that $\operatorname{occ}\left(y, U_{k}\right), \operatorname{occ}\left(y, V_{k}\right) \leq 1$ for all $y \in \Sigma$. Therefore it suffices to show that $\operatorname{occ}\left(y, U_{k}\right) \equiv \operatorname{occ}\left(y, V_{k}\right)(\bmod 2)$ for any $y \in \Sigma$. There are two cases to consider.

CASE 1: $x_{k}^{e_{k}}$ and $x_{k}^{f_{k}}$ are first stacks of $U$ and $V$ respectively. Then

$$
\begin{aligned}
\operatorname{occ}(\dot{y}, x, U) & =\operatorname{occ}\left(y, x_{0}^{e_{0}} \prod_{i=1}^{k-1}\left(U_{i} x_{i}^{e_{i}}\right)\right)+\operatorname{occ}\left(y, U_{k}\right) \\
\text { and } \operatorname{occ}(\dot{y}, x, V) & =\operatorname{occ}\left(y, x_{0}^{f_{0}} \prod_{i=1}^{k-1}\left(V_{i} x_{i}^{f_{i}}\right)\right)+\operatorname{occ}\left(y, V_{k}\right) .
\end{aligned}
$$

It follows from (B) and Lemma 2.3 (iii) that $\operatorname{occ}\left(y, U_{k}\right) \equiv \operatorname{occ}\left(y, V_{k}\right)(\bmod 2)$. CASE 2: $x_{k}^{e_{k}}$ and $x_{k}^{f_{k}}$ are last stacks of $U$ and $V$ respectively. Then

$$
\begin{aligned}
\operatorname{occ}(y, U) & =\operatorname{occ}\left(y, x_{0}^{e_{0}} \prod_{i=1}^{k-1}\left(U_{i} x_{i}^{e_{i}}\right)\right)+\operatorname{occ}\left(y, U_{k}\right)+\operatorname{occ}\left(y, x_{k}^{e_{k}} \prod_{i=k+1}^{m}\left(U_{i} x_{i}^{e_{i}}\right)\right) \\
& =\operatorname{occ}\left(y, x_{0}^{e_{0}} \prod_{i=1}^{k-1}\left(U_{i} x_{i}^{e_{i}}\right)\right)+\operatorname{occ}\left(y, U_{k}\right)+\operatorname{occ}\left(x_{k}, \dot{y}, U\right)
\end{aligned}
$$

and similarly,

$$
\operatorname{occ}(y, V)=\operatorname{occ}\left(y, x_{0}^{f_{0}} \prod_{i=1}^{k-1}\left(V_{i} x_{i}^{f_{i}}\right)\right)+\operatorname{occ}\left(y, V_{k}\right)+\operatorname{occ}\left(x_{k}, \dot{y}, V\right) .
$$

It follows from (B) and Lemmas 2.2 and 2.3(iv) that $\operatorname{occ}\left(y, U_{k}\right) \equiv \operatorname{occ}\left(y, V_{k}\right)$ $(\bmod 2)$.

Lemma 5.6. $U=V$.

Proof. By Lemmas 5.3-5.5,

$$
U=x_{0}^{e_{0}} \prod_{i=1}^{m}\left(U_{i} x_{i}^{e_{i}}\right) \quad \text { and } \quad V=x_{0}^{f_{0}} \prod_{i=1}^{m}\left(U_{i} x_{i}^{f_{i}}\right)
$$


where $m \geq 1$ and $e_{i} \equiv f_{i}(\bmod 2)$ for all $i$. Let $k \in\{0,1, \ldots, m\}$. It is shown in each of the following collectively exhaustive cases that $e_{k}=f_{k}$. Therefore the identity $U \approx V$ is trivial and is vacuously implied by the identities (0).

CASE 1: $x_{k}^{e_{k}}$ is a lone stack of $U$. Then $x_{k}^{f_{k}}$ is also a lone stack of $V$ by Lemma 5.1. Therefore $e_{k}, f_{k} \leq 14$ by (CF5)(a) with $e_{k}=\operatorname{occ}\left(x_{k}, U\right)$ and $f_{k}=\operatorname{occ}\left(x_{k}, V\right)$. If $e_{k} \in\{1,2\}$, then clearly $e_{k}=f_{k}$ by Lemma 2.1(i). If $e_{k} \in\{3,4, \ldots, 14\}$, then $f_{k} \in\{3,4, \ldots, 14\}$ by Lemma 2.1(ii), whence $e_{k}=f_{k}$ by Lemma 2.2 .

CASE 2: $x_{k}^{e_{k}}$ is a non-lone first stack of $U$. Then $x_{k}^{f_{k}}$ is also a non-lone first stack of $V$. Therefore $e_{k}, f_{k} \leq 2$ by $(\mathrm{CF} 5)(\mathrm{b})$, whence $e_{k}=f_{k}$ by Lemma 5.4. CASE 3: $x_{k}^{e_{k}}$ is a non-lone last stack of $U$. Then there exists some $h<k$ such that $x_{h}=x_{k}$; it is notationally simpler to write $x=x_{h}=x_{k}$. Hence

$$
U=\underbrace{x_{0}^{e_{0}}\left(\prod_{i=1}^{h-1}\left(U_{i} x_{i}^{e_{i}}\right)\right) U_{h}}_{A} \cdot x^{e_{h}}\left(\prod_{i=h+1}^{k-1}\left(U_{i} x_{i}^{e_{i}}\right)\right) U_{k} x^{e_{k}} \cdot \underbrace{\left(\prod_{i=k+1}^{m}\left(U_{i} x_{i}^{e_{i}}\right)\right)}_{B}
$$

where $x \notin \operatorname{con}(A B)$. It follows that there are two $x$-stacks in $V$. By Case 2, the exponent $e_{h}$ of the first $x$-stack of $U$ coincides with the exponent of the first $x$-stack of $V$. Therefore

$$
V=\underbrace{x_{0}^{f_{0}}\left(\prod_{i=1}^{h-1}\left(U_{i} x_{i}^{f_{i}}\right)\right) U_{h}}_{A^{\prime}} \cdot x^{e_{h}}\left(\prod_{i=h+1}^{k-1}\left(U_{i} x_{i}^{f_{i}}\right)\right) U_{k} x^{f_{k}} \cdot \underbrace{\left(\prod_{i=k+1}^{m}\left(U_{i} x_{i}^{f_{i}}\right)\right)}_{B^{\prime}}
$$

where $x \notin \operatorname{con}\left(A^{\prime} B^{\prime}\right)$. Let $p=e_{h}+\operatorname{occ}\left(x, \prod_{i=h+1}^{k-1} U_{i}\right)$, so that

$$
\operatorname{occ}(x, U)=p+e_{k} \quad \text { and } \operatorname{occ}(x, V)=p+f_{k} .
$$

There are two subcases.

Subcase 3.1: $p=1$. Then $\operatorname{occ}(x, U)=e_{k}+1$ and $\operatorname{occ}(x, V)=f_{k}+1$, so that $e_{k}, f_{k} \leq 13$ by $(\mathrm{CF} 5)(\mathrm{c})$. Hence $\operatorname{occ}(x, U), \operatorname{occ}(x, V) \in\{2,3, \ldots, 14\}$. If $e_{k}=1$, so that $\operatorname{occ}(x, U)=2$, then $\operatorname{occ}(x, V)=2$ by Lemma 2.1(i), whence $f_{k}=1=e_{k}$. Therefore it remains to assume that $e_{k} \in\{2,3, \ldots, 13\}$, so that $\operatorname{occ}(x, U) \in\{3,4, \ldots, 14\}$. Now $\operatorname{occ}(x, V) \in\{3,4, \ldots, 14\}$ by Lemma 2.1(ii), hence $e_{k}=f_{k}$ by Lemma 2.2 .

Subcase 3.2: $p \geq 2$. Then $\operatorname{occ}(x, U) \geq e_{k}+2$ and $\operatorname{occ}(x, V) \geq f_{k}+2$, so that $e_{k}, f_{k} \leq 12$ by $(\mathrm{CF} 5)(\mathrm{d})$. Since

$$
p+e_{k}=\operatorname{occ}(x, U) \equiv \operatorname{occ}(x, V)=p+f_{k} \quad(\bmod 12)
$$

by Lemma 2.2 , it follows that $e_{k}=f_{k}$.

ACKNOWLEDGEMENTS.

The authors are indebted to the anonymous reviewer for many helpful 
comments and suggestions, and to James D. Mitchell for providing, from GAP [3], the multiplication tables of monoids of order four in Section 3.

\section{REFERENCES}

[1] J. Almeida, Finite semigroups and universal algebra, World Scientific, Singapore, 1994.

[2] A. P. Birjukov, Varieties of idempotent semigroups, Algebra i Logika 9 (1970), 255-273 (in Russian); English transl.: Algebra and Logic 9 (1970), 153-164.

[3] A. Distler and J. D. Mitchell, Smallsemi - a GAP package, version 0.6.6, 2013, available at http://www.gap-system.org/Packages/smallsemi.html

[4] C. C. Edmunds, On certain finitely based varieties of semigroups, Semigroup Forum 15 (1977/78), 21-39.

[5] C. C. Edmunds, Varieties generated by semigroups of order four, Semigroup Forum 21 (1980), 67-81.

[6] C. F. Fennemore, All varieties of bands. I, II, Math. Nachr. 48 (1971), 237-252; ibid., 253-262.

[7] J. A. Gerhard, The lattice of equational classes of idempotent semigroups, J. Algebra 15 (1970), 195-224.

[8] M. Jackson, Finite semigroups whose varieties have uncountably many subvarieties, J. Algebra 228 (2000), 512-535.

[9] E. W. H. Lee, Hereditarily finitely based monoids of extensive transformations, Algebra Universalis 61 (2009), 31-58.

[10] E. W. H. Lee, Varieties generated by 2-testable monoids, Studia Sci. Math. Hungar. 49 (2012), 366-389.

[11] E. W. H. Lee, Finite basis problem for semigroups of order five or less: generalization and revisitation, Studia Logica 101 (2013), 95-115.

[12] E. W. H. Lee and J. R. Li, Minimal non-finitely based monoids, Dissertationes Math. (Rozprawy Mat.) 475 (2011), 65 pp.

[13] E. W. H. Lee and W. T. Zhang, Finite basis problem for semigroups of order six, LMS J. Comput. Math. 18 (2015), 1-129

[14] J. R. Li and Y. F. Luo, Equational property of certain transformation monoids, Internat. J. Algebra Comput. 20 (2010), 833-845.

[15] J. R. Li, W. T. Zhang, and Y. F. Luo, On the finite basis problem for the variety generated by all n-element semigroups, Algebra Universalis 73 (2015), 225-248.

[16] Y. F. Luo and W. T. Zhang, On the variety generated by all semigroups of order three, J. Algebra 334 (2011), 1-30.

[17] S. Oates and M. B. Powell, Identical relations in finite groups, J. Algebra 1 (1964), 11-39.

[18] P. Perkins, Bases for equational theories of semigroups, J. Algebra 11 (1969), 298-314.

[19] M. V. Sapir, Problems of Burnside type and the finite basis property in varieties of semigroups, Izv. Akad. Nauk SSSR Ser. Mat. 51 (1987), 319-340 (in Russian); English transl.: Math. USSR-Izv. 30 (1988), 295-314.

[20] L. N. Shevrin and M. V. Volkov, Identities of semigroups, Izv. Vyssh. Uchebn. Zaved. Mat. 1985(11), 3-47 (in Russian); English transl.: Soviet Math. (Iz. VUZ) 29(11) (1985), 1-64.

[21] The on-line encyclopedia of integer sequences, http://oeis.org/A058129

[22] A. N. Trahtman, Finiteness of a basis of identities of five-element semigroups, in: Semigroups and their homomorphisms (ed. E. S. Lyapin), Ross. Gos. Ped. Univ., Leningrad, 1991, 76-97 (in Russian).

[23] M. V. Volkov, The finite basis property of varieties of semigroups, Mat. Zametki 45 (1989), 3, 12-23 (in Russian); English transl.: Math. Notes 45 (1989), 187-194. 
[24] M. V. Volkov, The finite basis problem for finite semigroups, Sci. Math. Jpn. 53 (2001), $171-199$.

[25] M. V. Volkov, Reflexive relations, extensive transformations and piecewise testable languages of a given height, Internat. J. Algebra Comput. 14 (2004), 817-827.

E. W. H. Lee

Department of Mathematics

Nova Southeastern University

Fort Lauderdale, Florida 33314

USA

E-mail: edmond.lee@nova.edu

J. R. Li

School of Mathematics and Statistics

Lanzhou University

Lanzhou, Gansu 730000

P. R. China

E-mail: lijr@lzu.edu.cn

Received: 26.3.2014.

Revised: 18.1.2015. 